\title{
Novel Topical Microbicides Through Combinatorial Strategies
}

\author{
Anubhav Arora $\cdot$ Samir Mitragotri
}

Received: 10 January 2010 / Accepted: 15 February 2010 / Published online: 20 March 2010

(C) The Author(s) 2010. This article is published with open access at Springerlink.com

\begin{abstract}
Purpose Developing microbicides for topical epithelial applications is extremely challenging, as evidenced by the scarcity of approved products even after decades of research. Chemical enhancers, including surfactants, are known to be effective antimicrobial agents but are typically toxic towards epithelial cells. Here, we report on the discovery of unique surfactant formulations with improved safety and efficacy profile for epithelial applications, via a combination of high throughput screening techniques.
\end{abstract}

Methods Over three-hundred formulations derived from nine surfactants were screened for antibacterial properties against $E$. coli in vitro. A subset of these formulations showed high antibacterial activity and was screened for cytotoxicity in vitro. Formulations showing high antibacterial activity and reduced cytotoxicity compared to their individual components were tested for efficacy against $B$. thailendensis, a model for melioidosis-causing $B$. pseudomallei.

Results Lead formulations showed lower toxicity towards epidermal keratinocytes, with $\mathrm{LC}_{50}$ values up to 3.5-fold higher than their component surfactants, while maintaining antibacterial efficacy against $B$. thailendensis.

Conclusions Our results demonstrate that such a combinatorial screening approach can be used for designing safe and potent microbicides for epithelial applications.

KEY WORDS combinatorial · high throughput · microbicide safe $\cdot$ topical

A. Arora $\cdot$ S. Mitragotri $(\triangle)$

Department of Chemical Engineering,

Biomolecular Science and Engineering, University of California,

Santa Barbara, California 93106, USA

e-mail: samir@engineering.ucsb.edu

\section{INTRODUCTION}

Skin acts as a possible port of entry for numerous microorganisms. Human skin is regularly colonized by a number of non-pathogenic gram-positive bacteria, including different species of Staphylococci, Sarcina and Corynbacteria groups $(1,2)$. Gram-negative skin-resident bacteria are found in lesser abundance and include Mima, Herellea, Acinetobacter and Aerobacter. In addition, skin acts as a transient harbor to pathogenic bacteria or viruses to which it gets exposed. The topmost layer of skin, stratum corneum, provides a passive but formidable barrier to foreign entities and limits the habitat of these organisms to superficial layers. However, the barrier presented by stratum corneum can break down in the event of accidental cuts, bruises or pathological conditions, such as atopic dermatitis. The access to the underlying skin epithelium, epidermis, gives native and non-native skin flora the opportunity to infect the deeper layers of skin.

Epidermis is a highly potent immune organ and contains numerous resident cellular components of immune system, including keratinocytes, dendritic cells, mast cells, tissue macrophages and T lymphocytes $(3,4)$. Keratinocytes form the bulk of epidermal cellular population and orchestrate the innate immune response against invading pathogens by secreting pro-inflammatory cytokines and chemokines as well as by directly killing pathogens via secretion of antimicrobial peptides. The effectiveness of the epidermis in combating infections limits the number of skin infections, but does not completely eliminate them. Further, immunosuppressive conditions, such as atopic dermatitis, are coupled with high incidence of skin infections by bacteria and fungi, including Staphylococcus aureus, Staphylococcus epidermidis and Malassezia furfur (5).

As skin harbors several different strains of bacteria, frequent and uncontrolled use of antibiotics to treat pathological skin conditions increases the risk of emergence of antibiotic-resistant strains (6-8). Further, there is an increasing awareness regarding the need to sanitize skin for 
minimizing infections during invasive procedures, such as catheter insertion (9,10). Sanitization of skin and other body surfaces, such as nasal mucosa, will also prove highly beneficial during outbreaks of viral infections, such as influenza. Viral pathogens can have extended residence time on skin, thus increasing the likelihood of person-toperson transfer. Thus, there is a need to supplement the innate immune barriers with intelligently designed smallmolecule-based topical antimicrobial formulations as an alternative preventive measure.

Surfactants are one class of small molecules which have been well established as highly effective microbicides for the last several decades (11-13). Since the last decade, significant research and development efforts have been directed towards using nonionic surfactants as mucosal virucidal agents, but high levels of cytotoxicity continue to plague the current approach of using single surfactants $(14,15)$. The difficulty and complexity of discovering potent and safe microbicides is further evident from the fact that no safe microbicide has been developed for co-application on pathogens and viable human cells for any of the body surfaces.

Previously, we have successfully employed combinatorial high-throughput methods for finding unique combinations of small molecules, including surfactants, for needle-free transdermal drug and vaccine delivery (16-18). Here, those design principles are extended to the search of unique antimicrobial formulations which would be safe for application over large body surface areas. Using E. coli as a model gram-negative pathogen and human epidermal keratinocytes as a model epithelial cell line, a library of binary chemical formulations was screened for discovering unique combinations with high antibacterial efficacy and low toxicity.

\section{MATERIALS AND METHODS}

\section{Generation of Chemical Formulation Library}

Three surfactants were selected from each of the following chemical categories: (a) anionic surfactants, (b) cationic surfactants, (c) nonionic surfactants. The details, including full name, abbreviations used in this text and CAS registry numbers, are given in Table I. SLS, CPC, ALS, T20 and PEG were purchased from Sigma Aldrich (St. Louis, MO). CTC and BZK were purchased from TCI America (Portland, OR). TRL and S20 were purchased from Fisher Scientific (Fairlawn, NJ). Surfactant selection was primarily based on documented antibacterial activity and approval for usage in topical products by FDA, as per their GRAS (Generally Recognized as Safe) list status. All surfactants were paired with every other surfactant to form 36 binary pairs. Further, the weight fraction of components in each binary was varied from 0 to 1 , in steps of 0.1 each, to create 11 unique combinations. Thus, the total library consisted of 324 unique binary formulations. Each formulation was tested at total concentrations of $0.2 \%$, $0.1 \%, 0.05 \%$ and $0.025 \% \mathrm{w} / \mathrm{v}$. The value of total concentration was decided based on approved concentration limits for all the surfactants. Phosphate-buffered saline and glycerol mixed in equal volumes (1:1 PBS: Glycerol) was used as the universal solvent system for all formulations.

\section{Bacterial Strains, Growth Media and Culture Conditions}

Wild-type E. coli (strain ER2738) was purchased from New England Biolabs (Ipswich, MA) and was used as the model gram-negative pathogen. B. thailendensis (strain E264) was a gift from Dr. Peggy Cotter (UC Santa Barbara, CA). Leuria-Bertani (LB) broth $\left(10 \mathrm{~g}\right.$ tryptone $\mathrm{l}^{-1}, 5 \mathrm{~g}$ yeast extract $\left.l^{-1}, 10 \mathrm{~g} \mathrm{NaCl} \mathrm{l}^{-1}\right)$ made in ultrapure water and sterilized via autoclaving $\left(121^{\circ} \mathrm{C}, 15 \mathrm{~min}\right)$ was used for culturing E. coli. All components for making the LB broth were purchased from Fisher Scientific (Fairlawn, NJ). Precultures were prepared for each experiment by streaking stock solution (frozen in cryovials at $-80^{\circ} \mathrm{C}$ ) on $\mathrm{LB}$ agar plate. After overnight incubation of the plates at $37^{\circ} \mathrm{C}$, one colony was picked and loop-inoculated into a culture tube containing $5 \mathrm{ml} \mathrm{LB}$ broth. The culture tube was incubated $15-18 \mathrm{~h}$ at $37^{\circ} \mathrm{C}$ on a rotary shaker at $250 \mathrm{rpm}$. At the end
Table I List of Surfactants Used in this Study. Common Names, Abbreviations Used as Part of Text, Chemical Abstract Service (CAS) Registry Numbers, and Surfactant Category are shown

\begin{tabular}{lccc}
\hline \multicolumn{1}{c}{ Common name } & Abbreviation & CAS No. & Surfactant category \\
\hline Sodium lauryl sulfate & SLS & $|5|-2 \mid-3$ & Anionic \\
Ammonium lauryl sulfate & ALS & $2235-54-3$ & Anionic \\
Trolamine lauryl sulfate & TRL & $139-96-8$ & Anionic \\
Cetyltrimethylammonium chloride & CTC & $112-02-7$ & Cationic \\
Benzalkonium chloride & BZK & $139-08-2$ & Cationic \\
Cetylpyridinium chloride monohydrate & CPC & $6004-24-6$ & Cationic \\
Sorbitan monolaurate & S20 & $1338-39-2$ & Nonionic \\
Polysorbate 20 & T20 & $9005-64-5$ & Nonionic \\
Polyethylene glycol 200 & PEG & $25322-68-3$ & Nonionic \\
\hline
\end{tabular}


of incubation period, one-hundred micro-liters of this culture were transferred into a new culture tube containing $5 \mathrm{ml} \mathrm{LB}$ broth and grown to an $\mathrm{OD}_{600}$ value of 0.5 under the same incubation conditions. The $\mathrm{OD}_{600}$ cultures were diluted by a factor of $10^{3}$ in $\mathrm{LB}$ broth as working concentration and used immediately to minimize change in bacterial count. Low-sodium Leuria-Bertani (LSLB) broth $\left(10 \mathrm{~g}\right.$ tryptone $\mathrm{l}^{-1}, 5 \mathrm{~g}$ yeast extract $\left.\mathrm{l}^{-1}, 5 \mathrm{~g} \mathrm{NaCl} \mathrm{l}^{-1}\right)$ made in ultrapure water and sterilized via autoclaving $\left(121^{\circ} \mathrm{C}, 15 \mathrm{~min}\right)$ was used for culturing $B$. thailendensis. Culturing protocol was same as given above for $E$. coli.

\section{Screening for Antibacterial Efficacy Against E. coli}

Antibacterial efficacy of test formulations was quantified using BacTiter-Glo ${ }^{\mathrm{TM}}$ microbial cell viability assay (Promega Corp., Madison, WI) according to the protocols supplied by the manufacturer. The assay solution consisted of lysis buffer, beetle luciferin and recombinant firefly luciferase. Upon mixing the assay solution with bacterial culture, the bacterial cells were lysed, followed by extraction of ATP in the culture solution. Bioluminescence was produced as a result of reaction between beetle luciferin and recombinant firefly luciferase in the presence of ATP extracted from viable bacterial cells. The level of bioluminescence was directly proportional to the quantity of ATP present in the solution and was used for estimation of viable cells.

For the experiments, the E. coli cultures were prepared at working concentrations as described above and were dispensed in 96-well cell culture polypropylene plates (Corning, Lowell, MA) at $240 \mu \mathrm{l} /$ well. Inoculums in each well were incubated with $10 \mu \mathrm{l}$ of test formulations (at $25 \times$ concentration) for $4 \mathrm{~h}$ at $37^{\circ} \mathrm{C}$. Each formulation was tested at a final total concentration of $0.2 \%, 0.1 \%, 0.05 \%$ and $0.025 \% \mathrm{w} / \mathrm{v}$. Thus, the overall number of formulations tested was 1,296 or approximately 4,000 experiments including replicates. At the end of the incubation period, viable bacterial cells in each well were estimated using BacTiter-Glo ${ }^{\mathrm{TM}}$ assay.

\section{Minimum Inhibitory Concentration (MIC) Estimation in B. thailendensis}

Minimum inhibitory concentration against $B$. thailendensis was determined for select formulations. Broth microdilution method was followed for MIC determination, as per the protocol published by Wiegand et al (19). Briefly, fresh cultures were grown on the day of experiment as per the protocol described in "Bacterial Strains, Growth Media and Culture Conditions." The cultures were adjusted to $5.5 \times 10^{5} \mathrm{cfu} / \mathrm{ml}$ and used within $30 \mathrm{~min}$ to minimize change in bacterial counts. Cultures were dispensed in 96-well cell culture polypropylene plates (Corning, Lowell,
MA) at $90 \mu \mathrm{l} /$ well. Serial dilutions of test formulations were made at $10 \times$ concentration. Inoculums in each well were incubated with $10 \mu \mathrm{l}$ of test formulation dilutions for $18 \mathrm{~h}$ at $37^{\circ} \mathrm{C}$ under humidified conditions. At the end of incubation period, the plates were visibly inspected for bacterial growth. Colonies were counted for selected wells by plating culture dilutions on LSLB plates.

\section{Keratinocyte Cell Culture}

Primary epidermal keratinocyte cultures from an adult human source (HEKa) were purchased from Invitrogen Corp (Carlsbad, CA) and used for all cytotoxicity experiments. Cells were maintained in a humidified incubator $\left(37^{\circ} \mathrm{C}, 5 \% \mathrm{CO}_{2}\right)$ in EpiLife medium with $60 \mu \mathrm{M}$ calcium and phenol red, supplemented with $10 \mathrm{ml} / \mathrm{l}$ human keratinocyte growth supplement, $5 \mathrm{IU} / \mathrm{ml}$ penicillin and $5 \mu \mathrm{g} / \mathrm{ml}$ streptomycin. All components of growth media were purchased from Invitrogen Corp (Carlsbad, CA). Cells were grown to $70-80 \%$ confluence in cell culture flasks (Corning, Lowell, MA) as per suppliers' protocols.

\section{Screening for Cytotoxicity}

At the end of the growth period, keratinocyte cells were seeded at a density of $10^{4}$ cells/well in 96-well tissue culture treated polystyrene plates (Corning, Lowell, MA) and incubated overnight to allow cell attachment. Cells were supplied with fresh EpiLife medium (90 $\mu \mathrm{l} /$ well $)$ at the start of experiment, followed by application of test formulations $(10 \mu \mathrm{l} /$ well). The final concentration of test formulations in each well was $0.0001 \% \mathrm{w} / \mathrm{v}$. This concentration limit was determined based on the $\mathrm{LC}_{50}$ values of component surfactants for HEKa cell line, which were determined in a separate experiment. This concentration was used to maximize resolution between various surfactants and facilitate ranking of various formulations in terms of safety. The actual $\mathbf{L G}_{50}$ values in cells are likely to be much smaller than those in vivo, since under in vivo conditions, keratonocytes are expected to experience concentrations lower than those applied topically, since they are located farther away from the surface. Hence, the $\mathbf{L C}_{50}$ values should be used as an indication of relative safety and not absolute safety. The cells were incubated with the test formulations for $1 \mathrm{~h}$. At the end of the incubation period, culture medium was aspirated and replaced with $100 \mu \mathrm{l}$ of EpiLife medium without phenol red. Ten microliters of methyl thiazole tetrazolium solution $(5 \mathrm{mg} / \mathrm{ml})$ in phosphate-buffered saline was applied to each well for $4 \mathrm{~h}$, after which $100 \mu \mathrm{l}$ of acidified sodium lauryl sulfate solution $(10 \% \mathrm{w} / \mathrm{v}$ in $0.01 \mathrm{~N}$ hydrochloric acid) was added to each well. The plates were incubated for $16 \mathrm{~h}$ in a humidified environment, and absorbance was read at $570 \mathrm{~nm}$. 


\section{RESULTS}

Figure 1 shows the schematic of screening design used in this study. Three anionic, cationic and nonionic surfactants, which are approved for use in topical pharmaceutical applications, were used to design a combinatorial binary formulation library. The details for these chemicals are given in Table I. The current study focuses only on binary formulations and compares them to effectiveness and safety of single-surfactant formulations. All formulations were screened for antibacterial efficacy against E. Coli. Selected formulations, which showed significant antibacterial activity at the lowest concentration tested $(0.025 \% \mathrm{w} / \mathrm{v})$, were screened for cytotoxicity against epithelial cells. This concentration limit was primarily selected based on the approved concentration of these surfactants in the GRAS list. Binary formulations that exhibited higher $\mathrm{LC}_{50}$ values compared to that of both of their component surfactants were tested further for efficacy against $B$. thailandensis, a model pathogen for melioidosis causing $B$. pseudomallei.

Figure 2A shows the antibacterial efficacy of all the formulations tested in this study. The formulation index is an arbitrary number which identifies each formulation. Data shown are for each formulation at 4 different concentrations. Antibacterial potency (AP) represents loss of bacterial viability at the end of growth period, when the bacteria are grown in presence of test formulation, and is calculated as

$A P=\frac{V_{o}-V}{V_{o}} X 100$

where, $V$ is bacterial viability in the presence of test formulation, and $V_{o}$ is bacterial viability for negative control (bacterial viability without surfactant formulations).

The tested formulations showed a range of efficacy against $E$. coli with $36.3 \%$ completely suppressing the bacterial growth during the test period, while $7 \%$ had no significant effect on the growth (Fig. 2B). Approximately $38 \%$ were only mildly effective, with antibacterial activity of less than $25 \%$. A z-factor analysis was performed to assess the validity of the antibacterial screening assay. The z-factor was calculated to be 0.97 , confirming that the assay exhibits excellent screening qualities.

At a single surfactant level, the antibacterial activity was typically proportional to the surfactant concentration with cationic surfactants more potent than anionic surfactants, which were, in turn, more potent than nonionic surfactants. In binary combinations, the mixtures exhibited behavior depending on the type of surfactants (Fig. 3). Mixtures of anionic-anionic surfactants exhibited additive effects. For example, mixtures of a moderately potent anionic surfactant, SLS, with a weak anionic surfactant, ALS, led to
Fig. I High throughput screening design for discovery of safe and effective microbicides. Chemical enhancers were selected from FDA GRAS lists. Formulations were screened in vitro for antibacterial potential (AP) against E. coli as model gram negative pathogen and on adult human keratinocyte cultures for cytotoxicity

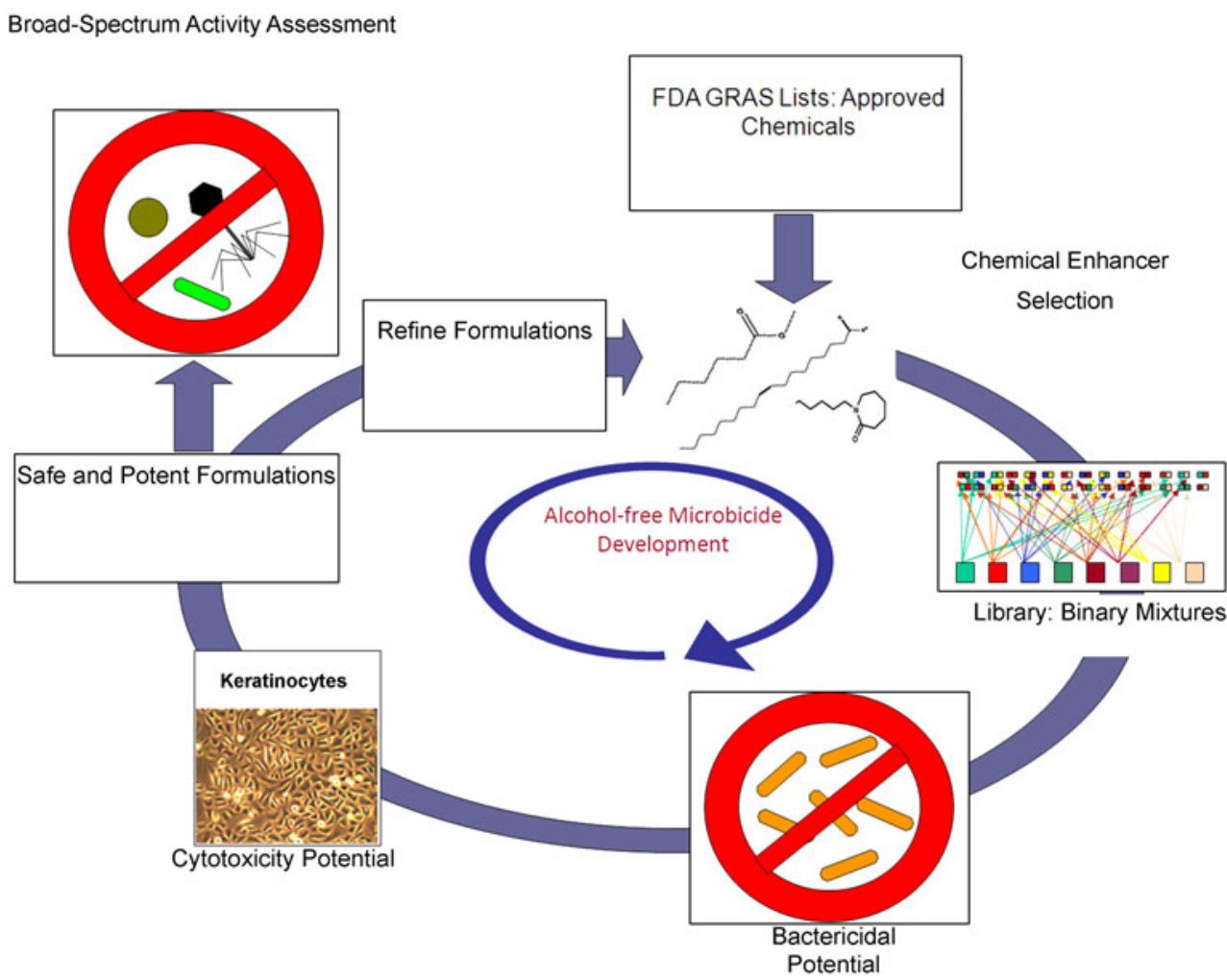




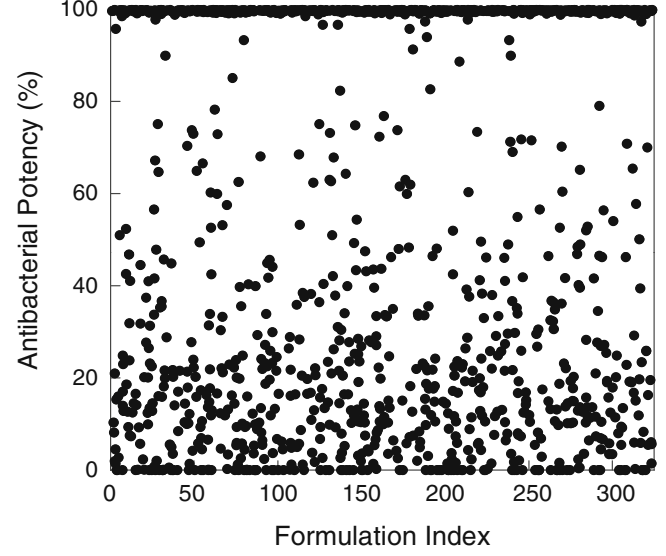

(A)

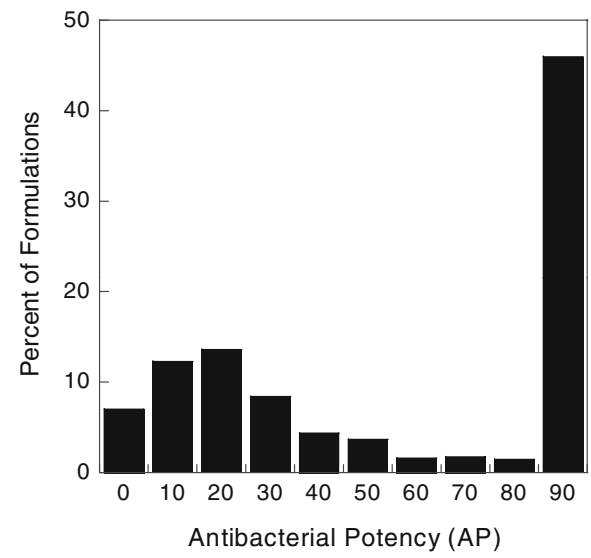

(B)

Fig. 2 Antibacterial activity of binary formulations. A Summary of antibacterial data obtained from binary formulations screened against E. coli using high throughout bioluminescent assay. Data shown are for 1296 formulations (324 unique binary formulations tested at 4 different total concentrations) and has been normalized with reference to negative controls. B Data from Fig. 2A binned to highlight the distribution of antibacterial potency. About 45\% formulations exhibited $>90 \%$ antibacterial potency. The rest of the formulations exhibit a range of antibacterial potencies with a peak at around $20 \%$.

formulations whose potency depended on the total SLS concentration (Fig 3A). The relationship of potency with the total SLS concentration in the formulation (open circles, Fig 3A) overlapped with that of pure SLS (closed circles, Fig 3A), suggesting that ALS acts merely as a "diluent" in this case. Similar trends were seen for mixtures of anionic and nonionic surfactants, for sample, SLS-S20 (Fig. 3B). Mixtures of anionic-cationic surfactants, for example, SLS-CTC (Fig. 3C), exhibited very different behavior. The activity of such mixtures did not exhibit a clear correlation with the concentration of SLS. This can be attributed to two reasons. First, in the case of SLS-CTC, the latter has higher antibacterial activity and hence dominates the behavior. Second, anionic and cationic surfactants interact strongly with each other, leading to unpredicted behavior. The data are also accompanied by high error. The error is high for equi-mass mixtures of SLSGTC. The experimentally observed error for equi-mass mixtures exceed $100 \%$ of the mean value, but drops to about $20 \%$ for mixtures where one of the ingredients is present in majority.

Mixtures of nonionic surfactants with other surfactants (Figs. 3D-F) did not exhibit any peculiar behavior. Owing to their relatively weak potency, the behavior of mixtures was dominated by the other component. Behavior of cationic surfactants (Figs. 3G-H), on the other hand, exhibited entirely opposite effect. Owing to their strong antibacterial activity, they dominated the behavior except when anionic surfactants were used in the mixture, which negated the potency of cationic surfactants due to charge neutralization.
It was generally observed that for a formulation to exhibit substantial antibacterial activity, at least one of the ingredients must possess antibacterial activity on its own. Further, the antibacterial activity of a formulation never exceeded that of its pure antibacterial components at the same concentration, thus showing a lack of synergy. This behavior is very different from that observed for skin permeation, where two permeation enhancers (including surfactants) often formed synergistic mixture that yielded significantly higher skin permeation enhancement compared to that of their individual components (16).

We next assessed whether mixtures of surfactants, though not capable of enhancing efficacy, are able to mitigate toxicity. Selected formulations which exhibited high antibacterial activity were tested for cytotoxicity (Fig. 4A). The formulations can be divided into five regions based on their antibacterial potency and toxicity behavior. Formulations in region I possess low potency and high toxicity. None of the formulations studied here belonged to this region. Formulations in region II are weakly potent but possess low cytotoxicity. Many binary formulations and a majority of individual enhancer (red circles) studies here belonged in this region. Region III corresponds to formulations with intermediate potency and toxicity. This region was relatively sparsely populated. Region IV corresponds to formulations with high potency and high toxicity. Region $\mathrm{V}$, the most desirable region, corresponds to high potency and low toxicity. Most individual chemicals were found to localize either in region II (low potency-low toxicity) or region IV (high potency-high toxicity). These data are in agreement with the widely reported limitations 


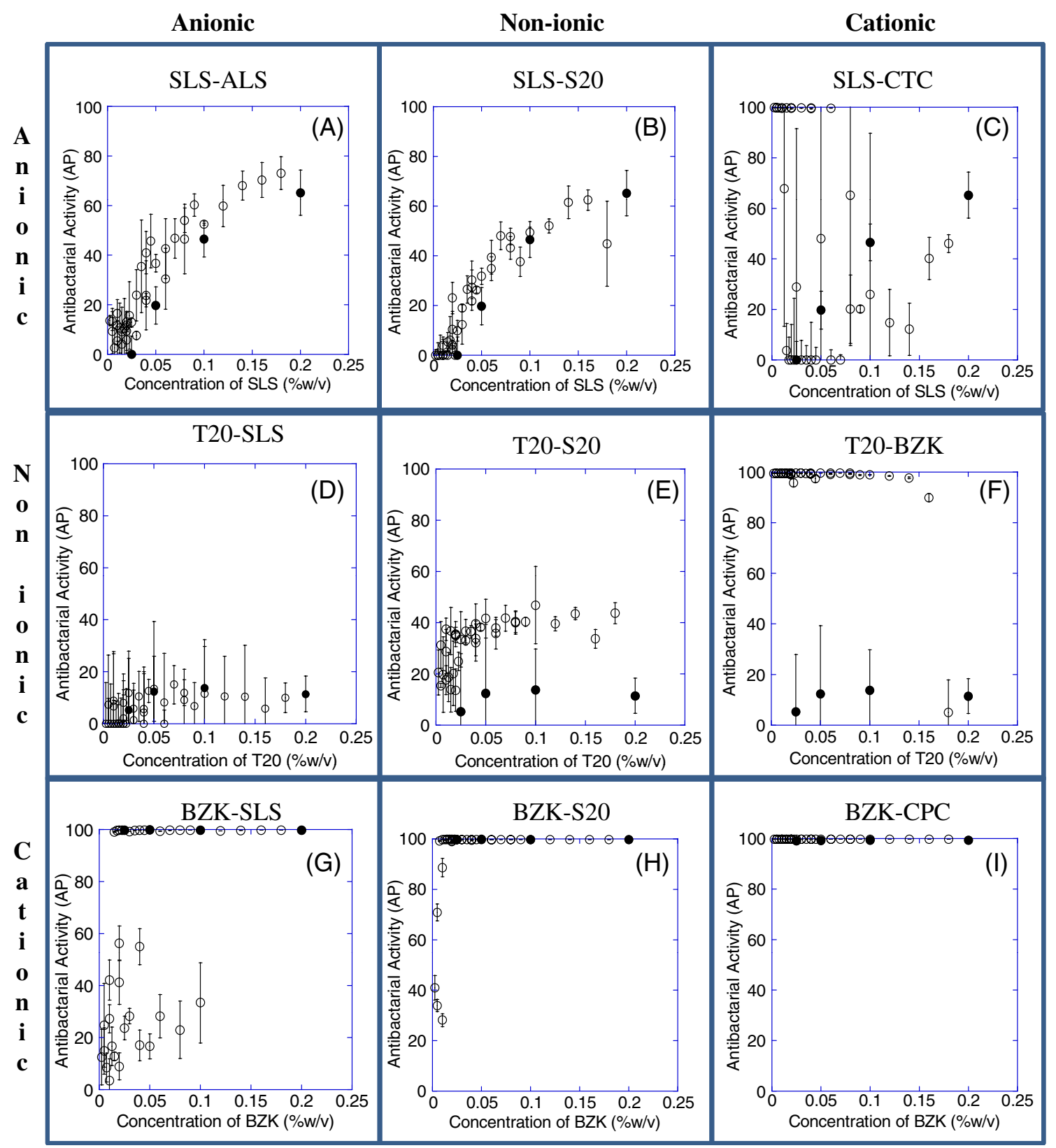

Fig. 3 Data describing antibacterial potencies of representative combinations of surfactants. A-C Combinations of SLS with three representative surfactants, each chosen from three categories of surfactants. D-F Combinations of T20 with three representative surfactants, each chosen from three categories of surfactants, G-I Combinations of BZK with three representative surfactants, each chosen from three categories of surfactants. Open circles indicate data for mixtures. Closed circles show data for pure surfactant (SLS for A-C, T20 for D-F, and BZK for G-I).

of single surfactants as microbicides and emphasize the advantage of combinatorial screenings. In contrast, binary formulations spanned a range of behavior, and some exhibited departure from expected correlation between efficacy and toxicity.

Formulations exhibiting high antibacterial potency $(>99 \%)$ and high keratinocyte viability $(>70 \%)$ were further analyzed. Interestingly, all these formulations comprised mixtures of BZK and S20. A detailed analysis of these mixtures revealed that antibacterial potency and cytotoxicity exhibited systematic dependence on the composition (Fig. 4B). S20 exhibited high cell viability but low antibacterial potency. BZK, on the other hand, exhibited high potency but low cell viability. Mixtures of BZK:S20 in the range of $30-70 \%$ BZK exhibited the ideal behavior. These formulations were tested for stability and potency against B. thailandensis. To further characterize their cytotoxicity, their lethal concentrations $\left(\mathrm{LC}_{50}\right)$ in keratinocytes were 


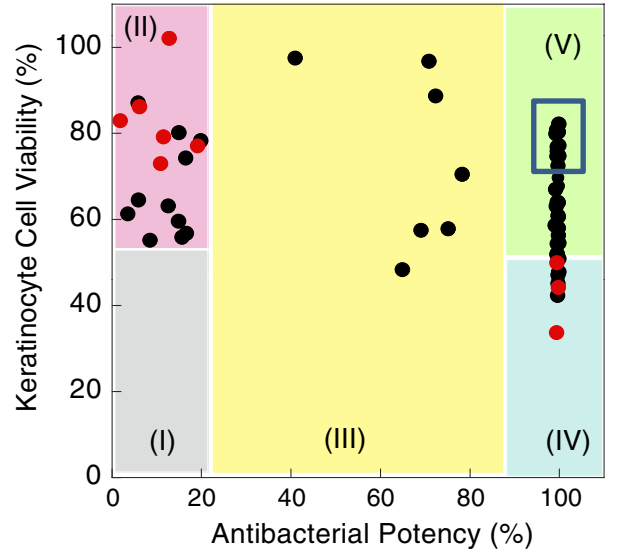

(A)

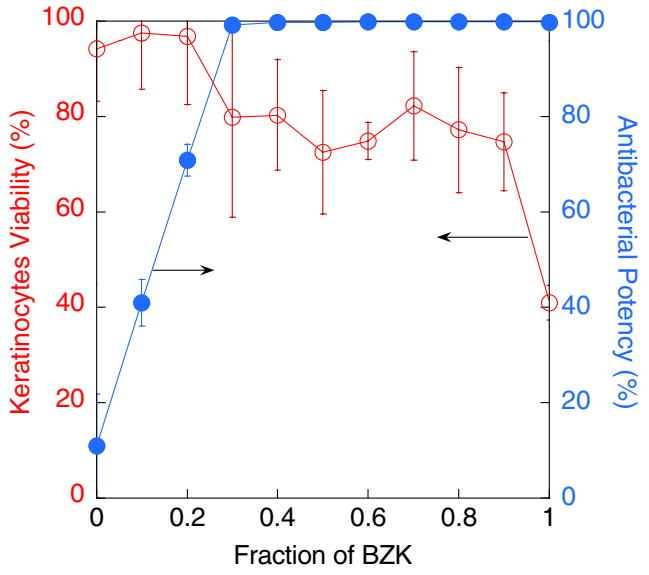

(B)

Fig. 4 Safety and efficacy of selected binary formulations. A Formulations showing high antibacterial potential against E. coli at lowest tested concentration $(0.025 \% \mathrm{w} / \mathrm{N})$ were tested for safety on human keratinocytes. Data distribution was divided into five distinct regions. Formulations in region I showed low safety and low potency. Formulations in region II showed high safety but low potency. Region III corresponds to formulations with moderate potency coupled with moderate to high safety. Formulations in region IV showed high potency and low safety. Formulations in region $V$ showed high potency coupled with moderate to high safety. Single surfactant data is shown for comparison (red circles). Cell viability data shown are for formulation concentration of $0.0001 \% \mathrm{w} / \mathrm{v}$. Antibacterial potency data shown is for formulation concentration of $0.025 \% \mathrm{w} / \mathrm{v}$. Concentrations were chosen for maximizing resolution in data sets. B Dependence of antibacterial potency and keratinocyte cell viability on the fraction of BZK in BZK:S20 formulations at a constant total concentration. Error bars represent standard deviation $(n>3)$.

determined. BZK exhibited low MIC $(0.00048 \% \mathrm{w} / \mathrm{v})$ and $\mathrm{LC}_{50}(0.00078 \% \mathrm{w} / \mathrm{v})$, whereas S20 exhibited negligible toxicity and potency in the range of concentrations studied. Binary compositions of BZK:S20 exhibited higher LC $_{50}$ values compared to BZK alone, indicating that addition of S20 to BZK decreases toxicity (Table II). However, addition of S20 also led to decreased potency as judged by increased MIC values. With two independent parameters (MIC and $\mathrm{LC}_{50}$ ), it is difficult to determine the benefits offered by binary formulations compared to single surfactant formulations. Therefore, the ratio of these two quantities $\left(\mathrm{LC}_{50} / \mathrm{MIC}\right)$ was used for determining the benefits of these formulations as potential microbicide (Fig. 5). The $\mathrm{LC}_{50} / \mathrm{MIC}$ ratios revealed that formulations of BZK and $\mathrm{S} 20$ exhibit up to 3-fold higher $\mathrm{LC}_{50} / \mathrm{MIC}$ ratio compared to $\mathrm{BZK}$ alone. Also, the $\mathrm{LC}_{50}$ values for all three formulations were higher than those of BZK $(p<0.05)$, proving their advantage as microbicides over application of BZK alone.

Table II List of MIC Values of Select Formulations Against B. thailandensis and $\mathrm{LC}_{50}$ Values in Keratinocytes

\begin{tabular}{ccc}
\hline Composition (BZK:S20) & MIC $\times 10^{4}(\% \mathrm{w} / \mathrm{v})$ & $\mathrm{LC}_{50} \times 10^{4}(\% \mathrm{w} / \mathrm{v})$ \\
\hline $4: 6$ & $>100$ & 2.83 \\
$5: 5$ & 15.63 & 2.04 \\
$6: 4$ & 9.38 & 1.89 \\
$7: 3$ & 9.11 & 1.80 \\
$10: 0$ & 7.48 & 0.48 \\
\hline
\end{tabular}

\section{DISCUSSION}

Several studies have reported on the use of various surfactants as potential microbicides (11-15). However, the associated toxicity has significantly thwarted the application of single-surfactant-based formulations. We have previously

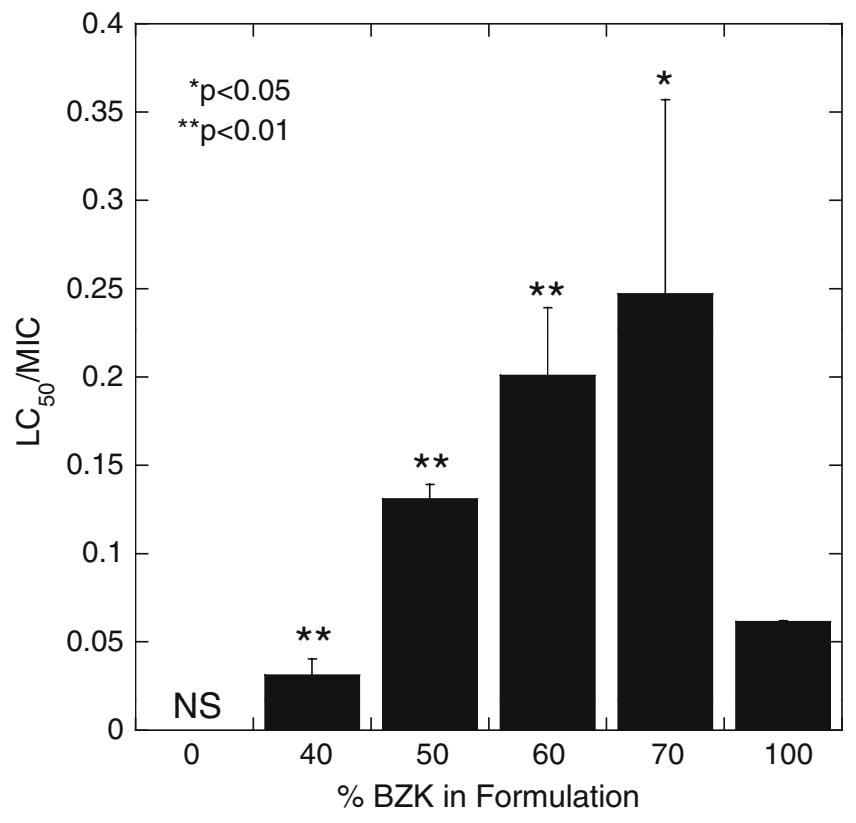

Fig. 5 Safety and potency of selected formulations. $\mathrm{LC}_{50} / \mathrm{MIC}$ values of selected BZK:S20 formulations. BZK is used as comparative control. Error bars represent standard deviation $(n=3)$. 
reported on combining chemicals, including surfactants, and using high-throughput screening for discovering combinations with desired properties for drug and vaccine delivery $(16,18)$. These desired properties included high skin permeation coupled with controlled toxicity. Results presented in this study extend the strength of combinatorial high-throughput screening to the discovery of surfactant formulations with high antimicrobial activity and controlled toxicity. Specifically, we investigated whether it is possible to discover surfactant combinations which are safer than single-surfactant formulations for application as epithelial microbicides.

Over 300 binary formulations were tested for their antimicrobial potency. More than one-third of the tested formulations completely suppressed bacterial growth under test conditions (Fig. 2), reaffirming the fact that antimicrobial potency is not the limiting step in microbicide development. Further, only those formulations which had surfactants exhibiting strong antibacterial activity by themselves were found to inhibit bacterial growth. This is a very interesting observation, since unlike skin permeation, no synergistic antimicrobial behavior was observed for any binary pair in the library studied. None of the formulations exhibited higher antimicrobial potency than their individual components. Chemicals from different chemical categories may be included in future screening designs for generalization of this observation. It should be noted that for most formulations, the concentrations of individual surfactants were within the FDA-approved limits for topical application, which increases the likelihood of such formulations being safe for human applications.

Data in Fig. 4A showed that a significant proportion of formulations were either safe but ineffective (region II) or potent but toxic (region IV). An equally significant fraction of formulations appeared to be strongly antibacterial, yet potentially safe for epithelial application. This was higher than the expected ratio, considering the scarcity of such formulations when using single surfactants. Closer examination revealed that all these formulations were comprised of mixtures of BZK and S20. The strong correlation between epithelial toxicity and antimicrobial nature of surfactants, or other small molecules, makes discovery of such formulations extremely challenging. In the absence of fundamental principles which can be used for designing new molecules or discovering formulations with the desired safety and efficacy characteristics, a high-throughput approach such as the one described here can serve as a powerful tool for developing such formulations as well as for collecting data which can aid in our understanding of the toxicity challenge. The low frequency of occurrence of safe and effective formulations (less than $1 \%$ of the total library in the current study) further emphasizes the importance of high-throughput designs.

The study reported here is the first step in establishing such combinatorial high-throughout screenings as a powerful way for discovering unique formulations with desir- able antimicrobial properties. The results discussed here may be specific to the model pathogen and epithelial cell line used in screening, but serve as a cost effective screening method. Skin is a complex epithelial tissue composed of several different types of cells. Keratinocyte cultures serve as a screen to compare relative safety of various formulations. However, the formulations discovered using such in vitro systems should be tested in vivo using animals and finally humans to establish safety and efficacy. Future studies will focus on screening formulations targeted towards specific pathogens of interest. The chemical library should also be expanded to include more chemical categories with history of topical applications, including fatty acids, esters and amines. Studies should also focus on developing an understanding of how surfactants interact with each other and with cell and bacterial membranes so as to fundamentally understand the mechanisms of synergy.

\section{CONCLUSIONS}

The results discussed here highlight a new approach to surfactant-based microbicide development for epithelial applications. A combination of commercially available assays was adapted for high-throughput screening of surfactant formulations, which resulted in discovery of highly potent antimicrobial formulations. A small fraction of screened formulations were proven to be safer than the widely reported approach of using single surfactants, which is often plagued by high toxicity. Lack of fundamental understanding of interactions between formulation components and epithelial tissue coupled with the low frequency of occurrence of such novel formulations necessitate the use of high-throughput screens such as the ones discussed here. Further, the surfactant concentrations employed in this study are within FDA-approved limits for most formulations, thus increasing the likelihood of success during in vivo trials. Future studies should focus on expanding the pool of chemicals tested to other chemical classes beyond surfactants and on validation of these results in vivo.

\section{ACKNOWLEDGEMENTS}

The authors thank Dr. Christopher McAllister, Dr. Cyril George, Tracy Hsu and Andrea Jani at UG Santa Barbara for insightful discussions and technical help with in vitro assay development.

Open Access This article is distributed under the terms of the Creative Commons Attribution Noncommercial License which permits any noncommercial use, distribution, and reproduction in any medium, provided the original author(s) and source are credited. 


\section{REFERENCES}

1. Noble WC. Skin bacteriology and the role of staphylococcus aureus in infection. Br J Dermatol. 1998;139:9-12.

2. Kligman AM. The bacteriology of normal skin. In: Maibach $H$, Hildick-Smith G, editors. Skin bacteria and their role in infection. New York: McGraw-Hill; 1965.

3. Pivarcsi A, Nagy I, Kemeny L. Innate immunity in the skin: How keratinocytes fight against pathogens. Curr Immunol Rev. 2005;1:29-42.

4. Boss JD. Skin immune system (sis). In: Boss JD, editor. Cutaneous immunology and clinical immunodermatology. 3rd ed. Boca Raton: CRC Press LLC; 2005.

5. Leung DYM, Bieber T. Atopic dermatitis. Lancet. 2003;361 (9352):151-60.

6. Bolognia JL, Edelson RL. Spread of antibiotic-resistant bacteria from acne patients to personal contacts - a problem beyond the skin? Lancet. 1997;350(9083):972-3.

7. Marchese A, Schito GC, Debbia EA. Evolution of antibiotic resistance in gram-positive pathogens. J Chemother. 2000;12 (6):459-62.

8. Van der Mee-Marquet $\mathrm{N}$ et al. Virulence and antibiotic susceptibility of staphylococcus aureus strains isolated from various origins. Pathol Biol. 2004;52(10):579-83.

9. Darouiche RO, Raad II. Prevention of catheter-related infections: the skin. Nutrition. 1997;13(4):S26-9.
10. Yentur EA et al. Is skin disinfection with $10 \%$ povidone iodine sufficient to prevent epidural needle and catheter contamination? Reg Anesth Pain Med. 2003;28(5):389-93.

11. Piret $\mathrm{J}$ et al. Comparative study of mechanisms of herpes simplex virus inactivation by sodium lauryl sulfate and n-lauroylsarcosine. Antimicrob Agents Chemother. 2002;46(9):2933-42.

12. Krebs FC et al. Inactivation of human immunodeficiency virus type 1 by nonoxynol-9, c31g, or an alkyl sulfate, sodium dodecyl sulfate. Antiviral Res. 1999;43(3):157-73.

13. Asculai SS et al. Inactivation of herpes-simplex viruses by non-ionic surfactants. Antimicrob Agents Chemother. 1978;13(4):686-90.

14. Niruthisard S, Roddy RE, Chutivongse S. The effects of frequent nonoxynol-9 use on the vaginal and cervical mucosa. Sex Transm Dis. 1991;18(3):176-9.

15. Roddy RE et al. A dosing study of nonoxynol-9 and genital irritation. International Journal of STD \& AIDS. 1993;4(3):165-70.

16. Karande P, Jain A, Mitragotri S. Discovery of transdermal penetration enhancers by high-throughput screening. Nat Biotechnol. 2004;22(2):192-7.

17. Karande P, Jain A, Mitragotri S. Insights into synergistic interactions in binary mixtures of chemical permeation enhancers for transdermal drug delivery. J Contr Release. 2006;115(1):85-93.

18. Karande $\mathrm{P}$, et al. Transcutaneous immunization using common chemicals. J Contr Release. 2009;138(2):134-40

19. Wiegand I, Hilpert K, Hancock REW. Agar and broth dilution methods to determine the minimal inhibitory concentration (mic) of antimicrobial substances. Nat Protocol. 2008;3(2):163-75. 